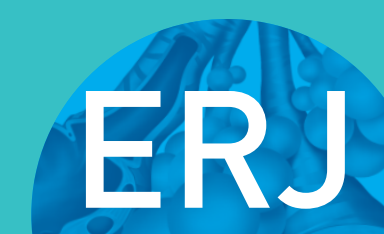

open research

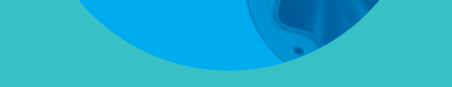

\section{German bronchoscopy unit readiness for the COVID-19 pandemic: a nationwide} survey

\author{
Clara S. Heidemann ${ }^{1,5}$, Jakob Garbe ${ }^{1,5}$, Marko Damm ${ }^{1}$, Steffen Walter ${ }^{2}$, \\ Patrick Michl ${ }^{1}$, Jonas Rosendahl ${ }^{1}$, Kaid Darwiche $\mathbb{1}^{3,4,5}$ and \\ Stephan Eisenmann (10 ${ }^{1,5}$
}

Affiliations: ${ }^{1}$ Dept of Internal Medicine I, University Hospital Halle, Halle, Germany. ${ }^{2}$ Dept of Medical Psychology, Clinic for Psychosomatic Medicine and Psychotherapy, University Hospital Ulm, Germany. ${ }^{3}$ West German Lung Center, Ruhrlandklinik, University Essen-Duisburg, Essen, Germany. ${ }^{4}$ German Respiratory Society (DGP), Berlin, Germany. ${ }^{5}$ These authors contributed equally.

Correspondence: Stephan Eisenmann, University Hospital Halle (Saale), Dept of Internal Medicine I, Ernst-Grube-Str. 40, 06120 Halle (Saale), Germany. E-mail: stephan.eisenmannđuk-halle.de

\section{ABSTRACT}

Background: The worldwide impact of the coronavirus disease 2019 (COVID-19) pandemic is unprecedented. Among the aerosol generating procedures, bronchoscopy in particular is an indispensable diagnostic and therapeutic tool that comes with a high risk of infection. Therefore, national societies have issued guidance statements. However, the individual ability of bronchoscopy units to follow these recommendations is largely unknown.

Methods: We conducted a nationwide survey from 1 April 2020 to 7 April 2020 to which 218 German endoscopy units, 105 solely bronchoscopy and 113 interdisciplinary, responded. The survey was distributed electronically via the German Respiratory Society.

Results: While $17 \%$ of units did not cancel any interventions, $16 \%$ cancelled $>80 \%$ of their interventions. $73 \%$ were unable to completely separate high-risk patients. Most procedural measures, such as risk stratification in patients (95\%), training to handle COVID-19 patients and personal protective equipment (PPE) (91\%), risk adapted use of PPE (85\%) and self-monitoring for staff (84\%) were adopted well. Unit managers expected shortages in PPE (74\%), staff shortages (68\%) and severe financial losses (63\%).

Conclusion: In the short-term, PPE shortages are perceived to be the most imminent threat to bronchoscopic activity as a whole. In this era of uncertainty, sound evidence to guide bronchoscopy units and an international concerted effort are urgently needed to formulate recommendations on facts and adapted to local conditions as described in this study.

@ERSpublications

Bronchoscopy units were partly prepared for the \#COVID19 pandemic. However, sound evidence to guide units and an international concerted effort are urgently needed to formulate recommendations for future pandemics. https://bit.ly/2B8WOPe

Cite this article as: Heidemann CS, Garbe J, Damm M, et al. German bronchoscopy unit readiness for the COVID-19 pandemic: a nationwide survey. ERJ Open Res 2020; 6: 00396-2020 [https:// doi.org/10.1183/23120541.00396-2020].

This article has supplementary material available from openres.ersjournals.com

Received: 18 June 2020 | Accepted: 22 June 2020

Copyright $\odot$ ERS 2020. This article is open access and distributed under the terms of the Creative Commons Attribution Non-Commercial Licence 4.0. 


\section{Introduction}

The coronavirus disease 2019 (COVID-19) pandemic is impacting healthcare systems worldwide on a hitherto unprecedented scale. Aerosol generating procedures are suspected to be a source of infection with severe acute respiratory syndrome-coronavirus-2 (SARS-CoV-2) for healthcare workers and patients. In this context, bronchoscopy is of particular significance as airway interventions are indispensable in the diagnosis and management of acute respiratory diseases. Yet, the aerosol generating procedure puts personnel at a special risk of infection [1].

To offer guidance in this situation, national societies, such as the German Respiratory Society (DGP), have issued recommendation statements [2]. These are based on expert opinions and the limited evidence available in this highly dynamic situation. However, little is known about the individual endoscopy units' structural and procedural ability to follow these recommendations. We conducted this survey to provide real-world data on German bronchoscopy units for further improvement of the regularly updated guidance statements.

\section{Methods}

In an expert panel, we derived questions on structural, procedural and personnel-related measures from the recommendations of the DGP and the European Society for Gastrointestinal Endoscopy [2, 3]. In addition, managers of endoscopy units were asked to provide individual approaches and their expectations for the future regarding personal protective equipment (PPE), shortages in staff and financial implications. Altogether, the survey contained 33 yes/no, multiple choice, Likert scale and free text questions. The full survey can be found in the supplementary material.

The local institutional review board approved the study (Versorgungsrealität in deutschen Endoskopiezentren in Zeiten der COVID-19-Pandemie, IRB no. 2020-044) and Good Clinical Practice standards were adhered to. The survey was conducted via the online survey tool LimeSurvey (www. limesurvey.org/en/), distributed electronically by the DGP and German Society of Gastroenterology, Digestive and Metabolic Diseases and addressed to endoscopy unit managers. The survey was open from 1 April to 7 April 2020, a phase of continued but slowed exponential growth in case numbers (figure 1).

In this article only data from respiratory medicine-oriented units are presented and discussed. The complete responses, including solely gastrointestinal endoscopy units, have been published in an abridged report elsewhere [5].

\section{Results}

Altogether, 107 bronchoscopy units answered our survey as well as 113 interdisciplinary units. The responses from two bronchoscopy units' were excluded as they were from outside Germany. Based on

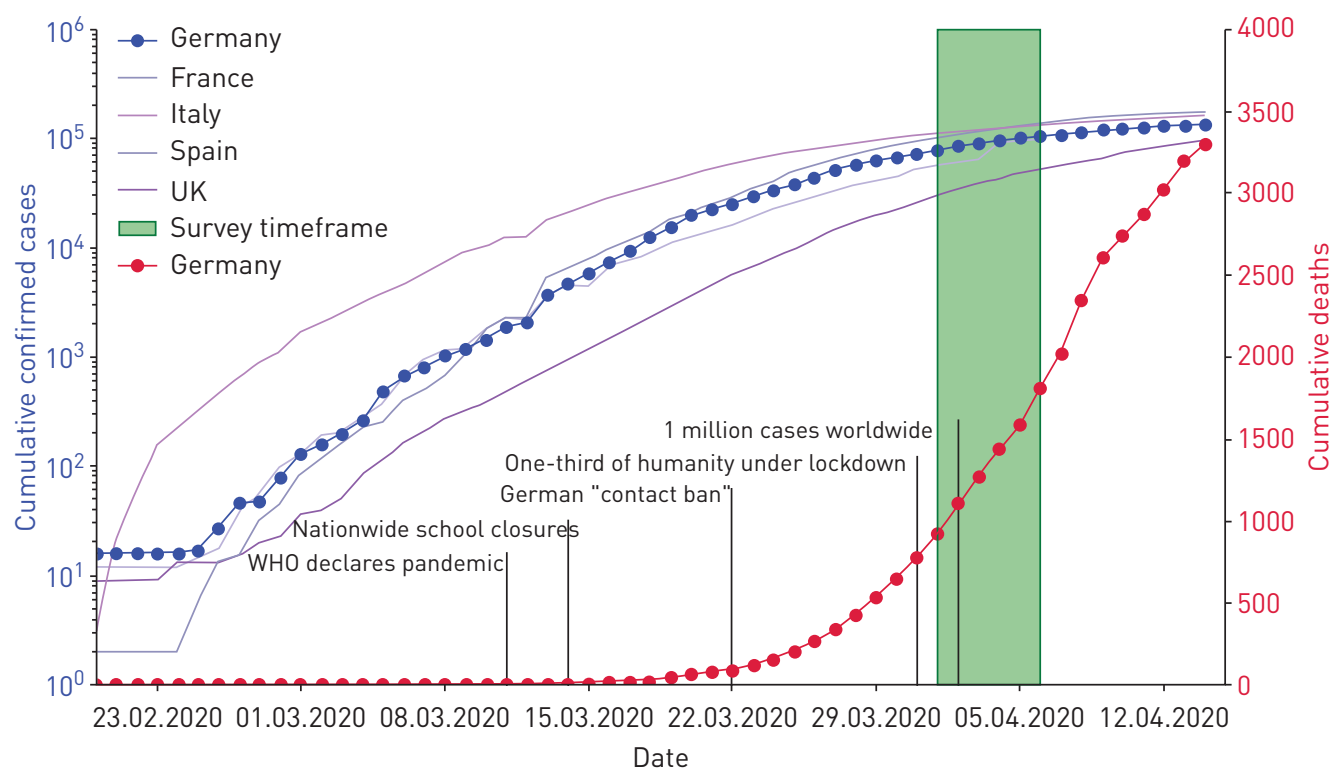

FIGURE 1 Survey timeframe in context of major events and progress of the coronavirus disease 2019 (COVID-19) pandemic. Neighboring European countries are plotted for comparison. For clarity, cumulative cases are plotted on a logarithmic scale. Data were retrieved from the Johns Hopkins University (Baltimore, MD, USA) [13]. 
FIGURE 2 Fraction of cancellations by unit size. Bronchoscopy units are divided into smaller units $1<2000$ procedures per year, $n=60$ ) and larger units (>2000, $\mathrm{n}=45) . \quad *$. fractions deviating $<5$ percentage points for combined data from interdisciplinary and bronchoscopy units $(n=218)$.

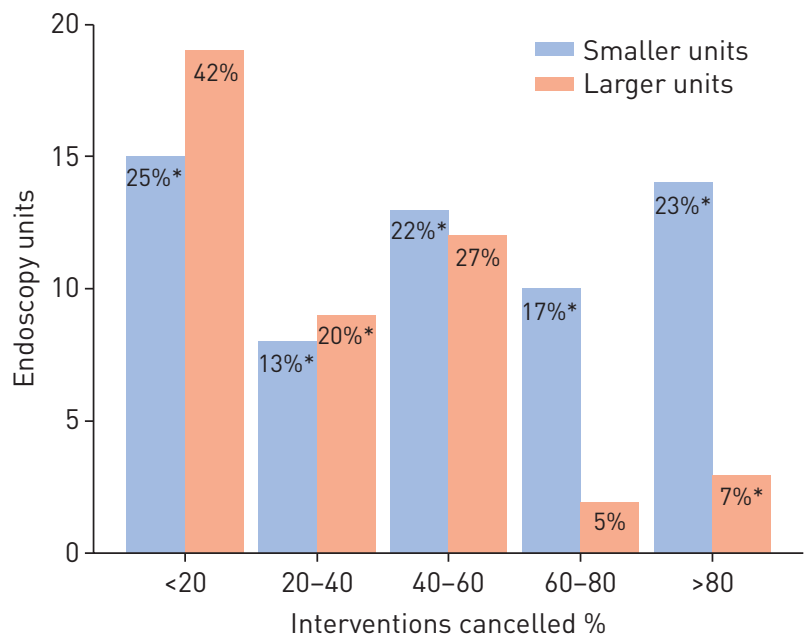

voluntarily provided postal codes, we screened for duplicates and did not identify any. For reasons of clarity we only show data from solely bronchoscopy units and indicate results that deviate $<5 \%$ for both interdisciplinary and bronchoscopy units with an asterisk $\left(^{*}\right)$. A full table of results is provided in the supplementary material.

Responses were spread all over Germany with a focus in the southern $\left(24 \%^{\star}\right)$ and eastern $\left(19 \%^{*}\right)$ regions (west $12 \%$, north $10 \%^{*}$ ), while $22 \%^{*}$ remained anonymous. The majority of responses came from hospitals $\left(26 \%^{*}\right.$ university or maximum care facility, $63 \%^{*}$ general or basic care) and $12\left(11 \%^{*}\right)$ out of 105 were from practices and outpatient clinics.

\section{Cancellations}

Fractions of cancelled interventions varied between endoscopy units and were distributed evenly along the spectrum (table S1). While $17 \% *$ of units did not cancel any interventions, $16 \%$ cancelled $>80 \%$ of their interventions. Particularly, practices and outpatient clinics cancelled $>80 \%$ of interventions (10 out of $12^{\star}$ ), in contrast to hospitals of any size $\left(7\right.$ out of $\left.93^{\star}\right)$. Smaller units $(<2000$ interventions per year) cancelled more interventions in relation to large $(>2000)$ units (figure 2$)$. Here, $40 \%{ }^{*}$ of smaller units cancelled $\geqslant 60 \%$ of interventions as opposed to $11 \%$ of larger units.

\section{Separation of high-risk patients}

$22 \%^{*}$ of units were able to completely separate proven or suspected COVID-19 patients. In addition, $5 \%^{*}$ were able to conduct procedures in a negative pressure room. In contrast, $42 \%$ could only partially (either endoscopy suite or peri-interventional) separate and 31\% were not able to separate at all (figure 3). $32 \%$ of hospitals were able to reserve separate equipment for COVID-19 patients. The size of units did not influence separability.

\section{Personnel}

$54 \% *$ of the units had a second team available if one had to move into quarantine. Half of the endoscopy managers also identified staff at risk for severe causes of COVID-19 and subsequently deployed these persons accordingly (low-risk area, administrative tasks, second team) in $77 \%^{\star}$ of cases. Routine swabs for staff were rarely conducted $\left(10 \%^{*}\right)$. In many cases $\left(81 \%^{*}\right)$ an infectiologist or hygiene specialist was

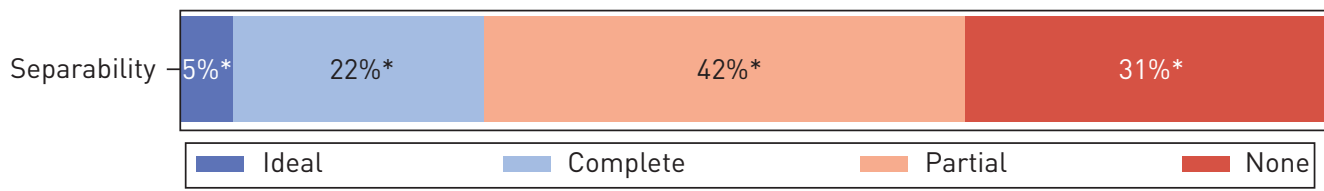

FIGURE 3 Spatial separability of high-risk patients within endoscopy units. Partial separability was given when either a separate endoscopy suite or separate peri-interventional area were available. For ideal conditions, units had to perform interventions in a negative pressure room in addition to complete separability. *: fractions deviating $<5$ percentage points for combined data from interdisciplinary and bronchoscopy units ( $n=218)$. 
consulted. Practices were restricted in their access to specialist consultations $\left(50 \%{ }^{\star}\right)$ as opposed to hospitals $\left(85 \%^{*}\right)$.

\section{Procedural measures}

Endoscopy units adopted procedural measures recommended to a varying degree. Structured risk stratification in patients $\left(95 \%^{*}\right)$, training to handle COVID-19 patients and PPE $\left(91 \% \%^{*}\right)$, risk adapted use of PPE $\left(85 \%^{\star}\right)$ and self-monitoring for staff $\left(84 \%^{\star}\right)$ were adopted well (figure 4 ). Almost half of units $\left(48 \%^{\star}\right)$ updated their instructions for the processing and cleaning of equipment. Pre-interventional swabs on patients were conducted in $32 \%^{\star}$ of units and a routine follow-up of patients was rare $\left(11 \%{ }^{\star}\right)$.

\section{Expectation for the future}

Regardless of unit size, expectations of endoscopy managers were predominantly negative: $74 \%^{*}$ expect shortages in PPE and $68 \%^{*}$ shortages in staff. Furthermore, 63\% supposed financial losses threatening the economic survival of the unit to be likely or very likely (figure 5). For practices and outpatient clinics, $93 \%{ }^{*}$ are expecting or already experienced shortages in PPE.

\section{Discussion}

On 18 March 2020, the DGP issued their guidance statement on the conduct of bronchoscopy during the COVID-19 pandemic for Germans [2], and other national societies have published further recommendations [6-9]. However, national guidelines differ considerable concerning recommendations for indications, use of PPE, optimal sedation regimen and other aspects, which reflects the limited evidence available in this highly dynamic situation [10].

National guidelines agreed on pre-interventional risk stratification of patients based on symptoms, sick contacts and vital signs [10]. According to our survey, this procedural measure was well adopted by endoscopy units. While risk stratification is undoubtedly needed, current evidence suggests that peak transmission is observed before the onset of symptoms [11]. Therefore, the assumed effectiveness of risk

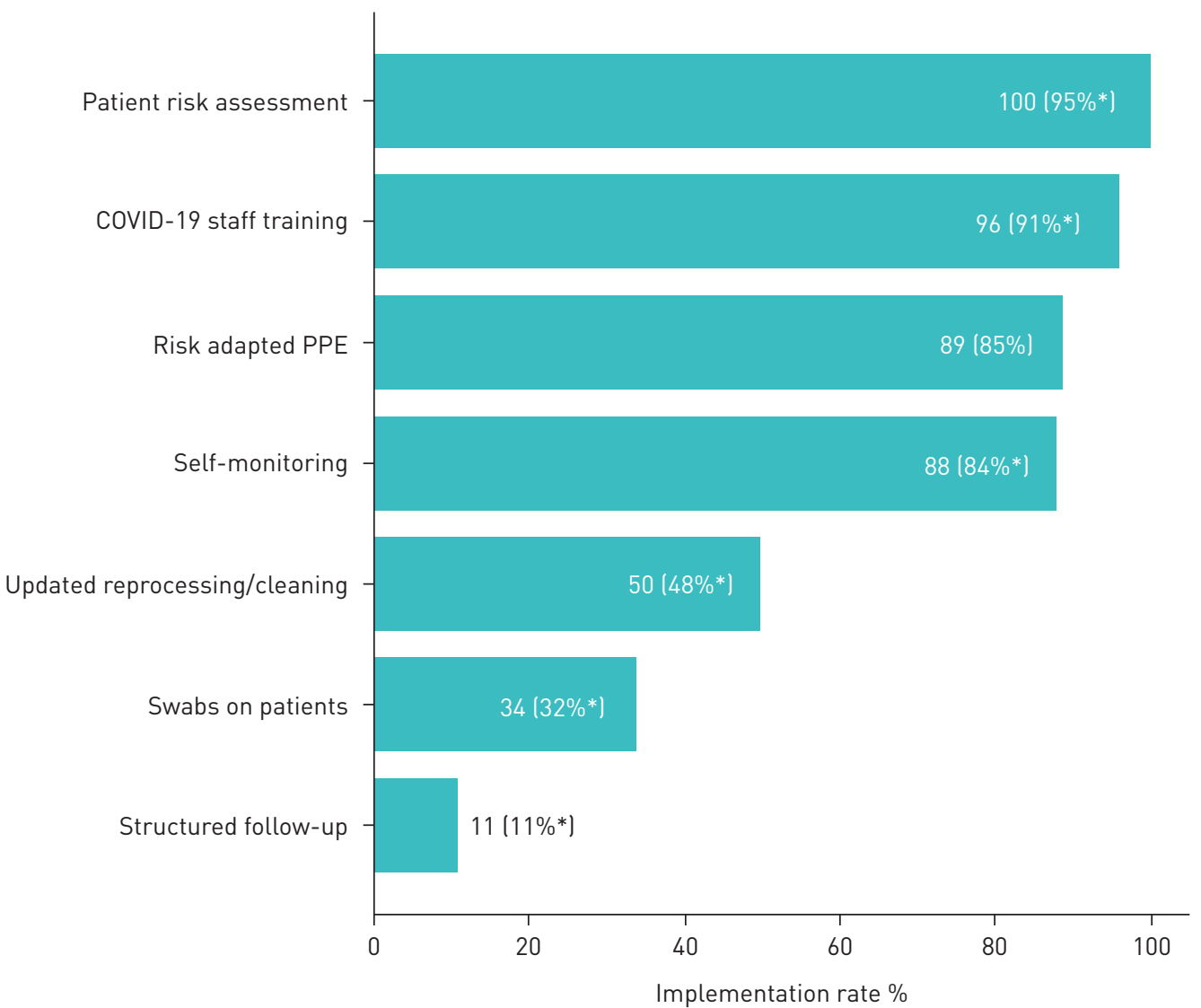

FIGURE 4 Implementation rates for procedural measures. COVID-19: coronavirus disease 2019; PPE: personal protective equipment. *: fractions deviating $<5$ percentage points for combined data from interdisciplinary and bronchoscopy units ( $n=218)$. 


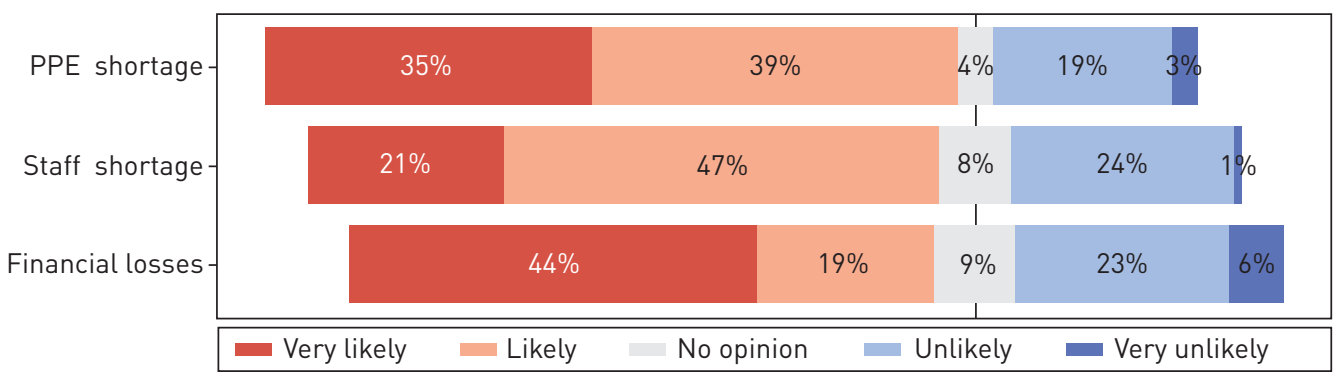

FIGURE 5 Expectations of managers of endoscopy units for the future. Diverging bar chart with neutral split indicated by vertical line. 5-step Likert scale for shortages in personal protective equipment (PPE), shortages in staff and financial losses threatening economic survival of the unit.

stratification protocols relying on symptoms and vital signs as a cornerstone might be limited. Consequently, protocols might be augmented by swabs for SARS-CoV-2 which were already implemented in one-third of units. However, availability, costs, latency of results and an estimated $20 \%$ false-negative rate might limit routine pre-interventional swabs as a viable strategy [12, 13].

Further procedural measures were adopted in most units except the routine follow-up of patients that is not mentioned in the DGP guidance. The survey questions concerning personnel were without corresponding items in the DGP statement either. However, many endoscopy managers identified personnel at risk for severe courses of COVID-19 and deployed these in low-risk areas. These common sense measures were implemented albeit missing sound evidence for their effectiveness. Also, the psychological burden for staff at risk working in a potentially high-risk environment must be acknowledged. Routine SARS-CoV-2 swabs for staff were a rarity. However, as pre-symptomatic transmission has to be considered, routine tests might be a reasonable strategy to avoid spreading the virus via endoscopy units without knowing.

A considerable fraction of interventions were cancelled in most units. Of note, $32 \%$ of units cancelled $<20 \%$ of procedures. Units with higher workload cancelled fewer interventions compared to lower workload units. It can be speculated that this is due to a greater fraction of hard to postpone procedures such as cancer diagnostics in larger units. The DGP statement left the decision as to which interventions to classify as elective or non-urgent in non-COVID-19 cases to endoscopy managers. While this practice allowed unit managers to adapt individual cancellations to local circumstances, the low cancellation rate in one-third of units suggests that endoscopic activity may not have been solely oriented on epidemiological concerns. In this context, the American Association for Bronchology and Interventional Pulmonology guideline might serve as a model, detailing which types of bronchoscopy are to be classified as non-urgent, urgent or emergency [7].

Endoscopy mangers stated different, yet, interlocking reasons for cancellations apart from DGP recommendations. These ranged from a lack of PPE to missing spatial separability of COVID-19 patients. Separation of high-risk patients was limited or impossible in $73 \%{ }^{*}$ of units. Only $5 \%$ had ideal structural conditions including a negative pressure endoscopy suite. As such, when planning endoscopy units in the future, structural conditions should allow for a short-term temporary spatial separation of patients.

Lack of PPE was a major concern for endoscopy managers as some declared to have ceased all endoscopic activity as a result of missing PPE. Accordingly, a shortfall in supply of bronchoscopy interventions might arise that would not only affect COVID-19 patients.

With responses from 218 interdisciplinary and solely respiratory medicine focussed endoscopy units, our survey most likely is representative of the nationwide endoscopic activity. However, the concrete number of German endoscopy units is not available to confirm this claim. Furthermore, $22 \%$ of unit managers remained anonymous and duplicates in these cases could not be ruled out.

We limited this survey to German endoscopy units for which the DGP guidance statement was issued. Transferability of findings from this survey to other healthcare systems or divergent epidemiological situations may, therefore, be limited. Finally, the questions of this survey were raised during an early period of the pandemic and critical points were already realised in many German hospitals.

\section{Conclusion}

In this unprecedented situation, endoscopy units quickly adapted to mitigate infection risk. While spatial separability of COVID-19 patients was limited in most units, procedural measures were adopted well. 
Pre-interventional risk stratification protocols may need to be updated, as symptom-based strategies are limited due to rising evidence of pre-symptomatic transmission. In addition, PPE shortages are perceived to be the most imminent threat to endoscopic activity. In this era of uncertainty, recommendations oriented on facts and local conditions as described in this study are urgently needed.

Acknowledgements: The authors thank all participating managers of endoscopy units for their responses, trust and insightful comments.

Author contributions: J. Garbe, S. Eisenmann and J. Rosendahl conceived, designed and directed the study. C.S. Heidemann, J. Garbe, S. Eisenmann, K. Darwiche and J. Rosendahl drafted and revised the manuscript with substantial help from P. Michl. J. Garbe, C.S. Heideemann and M. Damm performed statistical work and contentual review of survey responses. S. Walter guided the survey conception. All other co-authors helped to develop and disseminate the survey. All authors approved the final manuscript and contributed critical revisions to its intellectual content.

Data availability: Full data are provided in table S1.

Conflict of interest: None declared.

\section{References}

1 Tran K, Cimon K, Severn M, et al. Aerosol-generating procedures and risk of transmission of acute respiratory infections: a systematic review. Ottawa (ON), Canadian Agency for Drugs and Technologies in Health, 2011.

2 Darwiche K, Ross B, Gesierich W, et al. Recommendations for performing bronchoscopy in times of the COVID-19 pandemic. Pneumologie 2020; 74: 260-262.

3 Gralnek IM, Hassan C, Beilenhoff U. ESGE and ESGENA Position Statement on gastrointestinal endoscopy and the COVID-19 pandemic. Endoscopy 2020; 2020: 483-490.

4 Johns Hopkins University. Coronavirus resource center. https://coronavirus.jhu.edu/map.html Date last accessed: 15 April 2020.

5 Garbe J, Eisenmann S, Walter S, et al. German endoscopy unit preparations for the COVID-19 pandemic: A nationwide survey. Gastroenterology 2020; 159: 778-780.

6 Cordovilla R, Álvarez S, Llanos L, et al. Recomendaciones de consenso SEPAR y AEER sobre el uso de la broncoscopia y la toma de muestras de la vía respiratoria en pacientes con sospecha o con infección confirmada por COVID-19. Arch Bronconeumol 2020; 56: 19-26.

7 Wahidi MM, Shojaee S, Lamb CR, et al. The use of bronchoscopy during the COVID-19 pandemic: CHEST/ AABIP Guideline and Expert Panel Report. Chest 2020; in press [https://doi.org/10.1016/j.chest.2020.04.036].

8 AABE. [RECOMENDACIONES Asociación Argentina de Broncoesofagologia (AABE) basados en la Wold Association for Bronchology and Interventional Pulmonary WABIP para el manejo de pacientes con COVID 19 en situación de pandemia, 2020.000Z]. www.broncoscopia.org.ar/recomendaciones-asociacion-argentina-debroncoesofagologia-aabe-basados-en-la-wold-association-for-bronchology-and-interventional-pulmonary-wabippara-el-manejo-de-pacientes-con-covid-19-en-situacio/ Date last accessed: 18 May 2020.

9 Group of Interventional Respiratory Medicine, Chinese Thoracic Society. Expert consensus for bronchoscopy during the epidemic of 2019 Novel Coronavirus infection (Trial version). Zhonghua Jie He He Hu Xi Za Zhi 2020; 43: E006.

10 Lentz RJ, Colt H. Summarizing societal guidelines regarding bronchoscopy during the COVID-19 pandemic Respirology 2020; 25: 574-577.

11 He X, Lau EHY, Wu P, et al. Temporal dynamics in viral shedding and transmissibility of COVID-19. Nat Med 2020; 26: 672-675.

12 Ai T, Yang Z, Hou H, et al. Correlation of chest CT and RT-PCR testing in Coronavirus Disease 2019 (COVID-19) in China: a report of 1014 cases. Radiology 2020; 296: E32-E40.

13 Long C, Xu H, Shen Q, et al. Diagnosis of the coronavirus disease (COVID-19): rRT-PCR or CT? Eur J Radiol 2020; 126: 108961. 